\title{
Pasado y presente de los estudios del francés de especialidad
}

\section{Elena Cuasante Fernández ${ }^{1}$}

Universidad de Cádiz

\begin{abstract}
Over the last decades, specialised French has experienced some spectacular development as revealed by the appearance of numerous and very varied teaching methods. So today we find such a real conceptual galaxy of appellations and typologies that it is not always easy to orient oneself. For that reason, and with the aim of facilitating access to the current methodological range of specialised French, we have, in this study, accomplished both a revision of its main modalities and a delimitation of the teaching goals which characterise them.
\end{abstract}

Keywords: Specialised languages, FOS, FLP, FOU, FS.

\section{RESUMEN}

A lo largo de las últimas décadas, el francés de especialidad ha experimentado un desarrollo espectacular que se manifiesta en la aparición de numerosos y muy variados métodos de enseñanza. Así las cosas, nos encontramos hoy con una verdadera galaxia conceptual de apelaciones y tipologías en la que no siempre resulta fácil orientarse. Por ello, y con objeto de facilitar el acceso al actual panorama metodológico del francés de especialidad, en este estudio hemos llevado a cabo una revisión de sus principales modalidades, así como una delimitación de los objetivos docentes que las caracterizan.

Palabras clave: Lenguas de especialidad, FOS, FLP, FOU, FS.

1 Corresponding author - Universidad de Cádiz, Departamento de Filología Francesa e Inglesa, Avda. Gómez Ulla, 1, 11003 Cádiz (Spain).

Email: elena.cuasante@uca.es 


\section{Introduction}

En las últimas décadas, el debate alrededor de las diferentes modalidades de lo que habitualmente denominamos "francés de especialidad" se ha acelerado exponencialmente. Esto ha provocado que a metodologías ya existentes y consolidadas como el "Francés instrumental" o el "Francés funcional" se hayan sumado otras más recientes, entre las que se cuentan, por poner sólo algunos ejemplos, el "Francés Lengua Profesional", el "Francés para Fines Universitarios" o el "Francés Lengua de Integración". El indudable enriquecimiento que supone un incremento tan considerable no está exento, sin embargo, de cierta dosis de confusión, debida fundamentalmente a dos factores: por una parte, estamos ante un caleidoscopio terminológico y conceptual en el que no siempre es fácil orientarse; por otra, y como veremos más adelante, es un hecho que, aunque en principio remiten a competencias comunicativas, ámbitos de aplicación y colectivos de aprendizaje diferentes, las nuevas incorporaciones terminan por solaparse parcialmente. Este panorama nos sitúa frente a un desafío que Cadet y Guerin (2012. P. 3) han descrito muy acertadamente:

FLM (français langue maternelle) ou FL1 (français langue première), FLS ou FL2 (français langue seconde), FLE (français langue étrangère), FLSco (français langue de scolarisation), FOS (français sur objectifs spécifiques), FOA (français sur objectifs académiques), FOU (français sur objectifs universitaires), FLP (français langue professionnelle) et très récemment FLI (français langue d'intégration), autant de catégories créées autour de la langue française et de son enseignement, autant de lexies qu'il convient aujourd'hui de clarifier et d'interroger dans leurs rapports. Nombreux sont en effet les acteurs de l'enseignement du français qui cherchent à comprendre les raisons qui expliquent ce foisonnement terminologique, qui souhaitent définir les domaines que ces catégories délimitent, analyser la nature de leurs liens, le dessin des frontières, réelles ou supposées, qui les séparent, la façon dont elles dialoguent ou s'opposent. Démêler l'écheveau notionnel s'impose aujourd'hui : au regard de quoi, de qui, ces catégories sont-elles pertinentes?, sont-elles distinctes?, en tout ou en partie?

El presente estudio se enmarca precisamente en esta tarea, pues tiene como objetivo revisar cronológicamente las diferentes etapas que el francés de especialidad ha atravesado a lo largo de su evolución $y$, al mismo tiempo, reflexionar de manera distanciada sobre los aspectos teóricos y metodológicos que permiten definir sus diferentes modalidades.

\section{Desde de los orígenes hasta los años 80}

La historia del francés de especialidad, que remonta a los años 20 del siglo pasado, 
muestra claramente que la aparición de las sucesivas modalidades de enseñanza responde invariablemente a necesidades de aprendizaje específicas, relacionadas con el contexto histórico, social o económico en el que se enmarcan. Así, en 1927, en plena expansión colonial, se publica el que para la mayoría de los especialistas es el primer manual de francés para fines específicos: nos referimos al Règlement provisoire du 7 juillet pour l'enseignement du français aux militaires indigènes. Concebido para acercar a los soldados indígenas, cuya lengua materna no era el francés, al lenguaje militar, el texto constaba de 60 lecciones divididas en dos partes claramente diferenciadas. La primera contenía un vocabulario básico de palabras y expresiones propias de la vida castrense que se transmitían habitualmente con acompañamiento de imágenes y gestos y que, una vez adquiridas, permitirían a los oficiales blancos hacerse entender por la tropa: se trataba esencialmente de órdenes elementales, nombres de regimientos, tipos de municiones, etc. La segunda parte, publicada un año más tarde, ofrecía un arsenal semántico algo más prolijo, pues pretendía dotar a los soldados indígenas de competencias comunicativas suficientemente amplias como para que éstos se convirtieran en agentes activos del sistema colonial:

La guerre a mis en évidence les services que peuvent rendre les troupes indigènes et nous a laissé de nombreuses formations aptes à alléger, dans une grande mesure, les charges militaires qui, jusqu'alors, incombaient presque uniquement aux contingents de la Métropole. L'expérience a montré que, pour donner à ces troupes toute leur valeur, il était indispensable de leur assurer, dans la connaissance de notre langue, un bagage de plusieurs centaines de mots et d'expressions facilitant les rapports de la vie courante militaire et devant, d'ailleurs, après leur retour à la vie civile, contribuer au développement de la richesse de nos colonies en rendant plus aisées les relations des indigènes avec nos administrateurs, nos commerçants et nos industriels (cit. por Qotb, 2008 , p. 24) $)^{2}$.

A partir de los años 50-60, la enseñanza del francés de especialidad se orienta hacia un nuevo campo: el científico-técnico. Como es bien sabido, a lo largo de esta década Francia fue perdiendo gran parte de sus territorios africanos, lo que supuso un considerable descenso en el número de usuarios de la lengua francesa. A esta importante pérdida se sumaría además el auge del inglés, que también por estas fechas empieza a convertirse en la lengua de comunicación por excelencia. Así, a partir de los años 60, y con objeto de recuperar el terreno perdido, el gobierno galo intenta diversificar su presencia en el ámbito internacional por medio del "francés técnico y científico". Esta

2 Nos gustaría señalar la valiosa página web creada tras la defensa de su tesis por Hani Qotb sobre todos los aspectos relacionados con el francés de especialidad y que nos ha sido de gran utilidad para redactar el presente apartado de nuestro trabajo. Cfr. http://www.le-fos.com/. 
nueva orientación introduce algunos cambios significativos, pues aunque la adquisición de un léxico especializado sigue siendo un objetivo prioritario, antes de llegar a este punto los alumnos debían formarse en un denominado "francés general". La enseñanza quedaba así organizada en cuatro etapas: las dos primeras para la adquisición del francés básico o general, y las dos siguientes para la incorporación del vocabulario específico de un determinado campo de estudio ${ }^{3}$. Otra novedad importante fue la introducción de la metodología SGAV (structuro-global audiovisuelle), basada como sabemos en la combinación de la imagen y del sonido para la adquisición de la lengua.

Una década más tarde aparece en el panorama educativo el "francés instrumental", destinado, tal como queda recogido en la definición de Aupècle y Álvarez (1977), a un público de estudiantes universitarios que necesitaban comprender y elaborar escritos académicos: "C'est l'enseignement du français, langue étrangère, à des étudiants qui, sans se spécialiser en français, doivent avoir accès, en général dans leur pays, à des documents écrits de caractère informationnel" (1977, p. 101). Se trataba fundamentalmente de desarrollar la capacidad de lectura global del alumno, por lo que no se insistía excesivamente en trabajar contenidos relacionados con las necesidades específicas del alumno (cfr. Zolana, 2013, p. 29). El francés instrumental fue objeto de no pocas críticas por parte de los especialistas, que no comprendían que se dejasen de lado aspectos tan determinantes en la adquisición de una lengua como la comprensión y la expresión orales.

Pero será a finales de los 70 cuando se produzca un giro decisivo en lo que hoy concebimos como las lenguas de especialidad. El gobierno francés, muy afectado por la grave crisis del petróleo que se vivió en estos años, decidió orientar su política de expansión lingüística, y por ende económica, hacia otros públicos que no fuesen únicamente el compuesto por universitarios interesados en la carrera académica ${ }^{4}$. En esta línea, el Ministerio de Relaciones Exteriores de Francia crea diferentes centros de estudios y de documentación técnica y científica, así como un programa de becas titulado "Formation des boursiers prioritaires", dirigido exclusivamente a países no francófonos con relaciones estratégicas con el hexágono ${ }^{5}$. Nace así el francés funcional, que Porcher

3 Este giro en la enseñanza no hubiese sido posible sin el apoyo de instituciones políticas y del mundo de la edición que colaboraron en la publicación de diferentes manuales de léxico de especialidad denominados "VGOS" (Vocabulaire Général d'Orientation Scientifique) y de diccionarios de especialidad (Cfr. Qotb, 2008, pp. 31ss).

$4 \quad$ Cfr. http://www.le-fos.com/historique-4.htm

5 Se trataba de 9 países ricos en petróleo y en uranio, lo que viene a poner una vez más de manifiesto hasta qué punto la difusión del francés dependía de condicionantes exclusivamente 
definirá en 1976 en términos de gran simplicidad: "un français qui sert à quelque chose par rapport à l'élève" (1976, p. 13), o lo que es lo mismo, "celui qu'on enseigne en fonction d'un but" (Ibid). En cuanto a la metodología, en el ámbito del francés funcional se han ido proponiendo modelos diferentes, entre los que destacan el de Lehmann (1993), que primaba las necesidades de los alumnos, las situaciones de comunicación de cada sector y la expresión oral; el de Moirand (1993), que añade el análisis del discurso a los tres aspectos anteriores y, finalmente, el modelo curricular elaborado por Lehmann (1996) otra vez, en el que se insiste sobre los cuatro aspectos que rodean el aprendizaje, a saber: las características de los participantes, la situación de comunicación, el proyecto formativo y el análisis de documentos reales. A pesar de sus diferencias, estos tres modelos coinciden en su rechazo a los métodos basados en la simple adquisición del léxico (caso del francés militar), a los cursos demasiado extensos (caso del francés científico y técnico) y a la tendencia al universalismo que impregnaba la mayoría de las metodologías anteriores. Por último, conviene señalar que el francés funcional tampoco escapó a las críticas de quienes afirmaban, por ejemplo, que el análisis de las situaciones comunicativas no bastaba para completar el objetivo del aprendizaje, o que era un error dejar de lado las especialidades culturales de la lengua, indispensables en el intercambio profesional ${ }^{6}$. En cualquier caso, es hoy una verdad unánimemente reconocida que la aportación más decisiva del francés funcional consistió en tomar como punto de partida la consideración de las necesidades de los alumnos, elemento sobre el que se han construido igualmente todas las incorporaciones posteriores.

\section{De los años 80 a la actualidad}

El panorama que hemos descrito hasta aquí resulta verdaderamente sencillo si lo comparamos con el que se presenta posteriormente. A partir de los años 80, los estudiosos empiezan a tomar conciencia de que el francés de especialidad remite a objetivos y ámbitos de aplicación muy diversos que están por explorar, lo que origina un proceso de expansión verdaderamente espectacular. Desde entonces, nuevas tipologías y nomenclaturas se han ido sucediendo sin cesar, hasta conformar un extensísimo mosaico conceptual que Sowa y Gajewska (2013, p. 21) ilustran por medio de una tabla cronológica que reproducimos aquí parcialmente:

políticos. De hecho, la falta de presupuesto provocada por la segunda crisis del petróleo fue la principal causa del cese de estas becas y del fin del francés funcional.

$6 \quad$ En este sentido pueden evocarse los diferentes trabajos de Edward T. Hall y Mildred Reed Hall, y en particular Understanding Cultural Differences (1990), en los que se defiende que la comunicación no se hace únicamente a través de la lengua, sino también a través de otros elementos diferenciales de la cultura como la gestualidad. 


\begin{tabular}{ll}
\hline Appellation & Période \\
\hline Français sur objectif(s) Spécifique(s) (FOS) & $1980-$ \\
\hline Français de spécialité (FS) & $1990-$ \\
\hline Français de la communication professionnelle/ & $2000-$ \\
Français à visée professionnelle & \\
\hline Français langue professionnelle & $2006-$ \\
\hline Français langue d'action professionnelle & $2008-$ \\
\hline
\end{tabular}

Tabla 1. Cronología de términos y periodos asociados al francés de especialidad.

El principal inconveniente de tan súbita eclosión nominativa es la aparición de no pocos espacios de indefinición. En este sentido, no es casual que la bibliografía más reciente sobre el francés de especialidad esté constituida por estudios que reclaman una reflexión profunda sobre las fronteras que separan sus diferentes modalidades, entre los que pueden evocarse: "Français de spécialité ou français objectif spécifique: deux démarches didactiques distinctes" (Mangiante, 2006) ; "Du français sur Objectifs spécifiques au français Langue Professionnelle" y "Penser le français langue professionnelle" (MourlhonDallies, 2006); "Le FOS est-il soluble dans le FLE ?" (Richer, 2007); "La langue de spécialité est-elle soluble dans le FLE, le FOS et le FLP ?" (Gueorguieva-Steenhoute, 2008) o el "FS, FOS, FLP... Étiquettes vides ou concepts éducatifs".

En efecto, el principal desafío que presenta hoy el francés de especialidad es el de la delimitación de sus variantes, espinosa cuestión sobre la que intentaremos arrojar un poco de luz en las páginas que siguen.

El primer episodio de lo que podemos considerar como el reordenamiento conceptual del francés de especialidad nace con la llegada al panorama de la enseñanza del $\mathrm{FOS}^{7}$ Français sur Objectif(s) Spécifique $(\mathrm{s})^{8}$. Así lo defínía Cuq en su Dictionnaire de didactique du français langue étrangère et seconde : "Le français sur objectifs spécifiques (FOS) est né du souci d'adapter l'enseignement du Français langue étrangère (désormais FLE) à des publics adultes souhaitant acquérir ou perfectionner des compétences en français pour

7 Esta denominación es un calco del sintagma inglés "English for Specifics Purposes" que da nombre a un manual de Hutchinson y Waters (1987) en el que aparecen descritos por primera vez los principios fundamentales de las lenguas de especialidad. Su arraigo en el dominio educativo francés data de los años 1990 y 2004, cuando la revista Le Français dans le Monde, medio de difusión por excelencia de lo relativo a la lengua francesa, publica varios artículos con la expresión FOS en sus títulos.

8 La razón que justifica la inclusión entre paréntesis de la (s) es que, para muchos, la expresión "Français sur objectif(s) spécifique(s)" debe escribirse en singular, pues como veremos más adelante tiende a cubrir una demanda única. 
une activité professionnelle ou des études supérieures" (2003, p. 109)9.

La definición de Cuq establecía una clara diferenciación entre la enseñanza de lengua extranjera para un público generalista (FLE) y la enseñanza del francés para adultos inmersos ya en el mundo laboral o académico. Tampoco resultará especialmente complejo discriminar entre FOS (Français Objectif(s) Spécifique(s)) y FS (Français de Spécialité), expresión que aparece casi al mismo tiempo. Según Mangiante y Parpette (2004, p. 10), el FS y el FOS obedecen a situaciones o necesidades diferentes:

[...] celle qui relève de l'offre et celle qui relève de la demande. La première est une approche globale d'une discipline ou d'une branche professionnelle, ouverte à un public, le plus large possible. Elle tente de rendre compte de la diversité du champ traité. [...] Le FOS, à l'inverse, travaille au cas par cas, ou en d'autres termes, métier par métier, en fonction des demandes et des besoins d'un public précis.

Aquí también la diferencia reside en el tipo de público: si el enfoque se centra sobre una especialidad en su totalidad, sin que el público al que vaya destinado posea un conocimiento previo de la misma, hablaremos de "Français de Spécialité" (FS); si por el contrario, el programa de formación se dirige a un público ya especializado y con una demanda precisa de formación, estaríamos ante una metodología de "Français Objectifs Spécifiques" (FOS). Dos años más tarde Jean Marc Mangiante propone una tabla explicativa, que traducimos aquí de manera parcial, sobre el particular en la que se incluyen igualmente otros aspectos didácticos (2006, p. 142):

\begin{tabular}{ll}
\hline Francés de especialidad (FS) & $\begin{array}{l}\text { Francés para fines específicos } \\
\text { (FOS) }\end{array}$ \\
\hline Objetivo amplio sobre un dominio & $\begin{array}{l}\text { Objetivo más preciso sobre un } \\
\text { dominio }\end{array}$ \\
\hline $\begin{array}{l}\text { Formación a medio o a largo } \\
\text { plazo }\end{array}$ & Formación a corto plazo \\
\hline $\begin{array}{l}\text { Diversidad de temas y de } \\
\text { competencias ligadas a una } \\
\text { disciplina (economía, comercio, } \\
\text { medicina) }\end{array}$ & $\begin{array}{l}\text { Centrado en una situación de } \\
\text { comunicación específica }\end{array}$ \\
\hline
\end{tabular}

Tabla 2. Fragmento de la tabla ofrecida por Mangiante sobre las diferencias entre el francés de

9 Este mismo año Beatrice Tauzin propone una definición muy similar: "Le FOS ce n'est pas d'enseigner le français ou apprendre le français, mais c'est bien d'apprendre du français "pour". C'est du français pour travailler -pour les uns- et pour suivre des études -pour les autres" (2003, p. 82). 
especialidad y el francés para fines específicos.

El panorama estaba pues bastante claro hasta 2006, año en el que Mourlhon-Dallies (2006) introduce una nueva variable, la del "Français langue profesionnelle" (FLP), en cuya definición toma en consideración no sólo la formación y los objetivos del público, sino también su origen:

FLP est le français enseigné à des personnes devant exercer leur profession entièrement en français. [...] Cela différencie le FLP du FOS, car le FOS à un champ d'action beaucoup plus restreint : tel qu'il s'est développé depuis trente ans, le FOS permet essentiellement à des non natifs de maîtriser le français dans leur contexte professionnel d'origine, en ciblant des compétences limitées (besoins de lecture technique, contacts avec une clientèle française, négociations en français). On remarquera enfin que le FLP s'adresse pour partie à des natifs, relevant alors du français langue maternelle (FLM). Cette voie est jusqu'à présent peu explorée (2006, p. 30).

Empleado para distinguir el FLP (Français Langue Professionnelle) del FOS (Français Objectitf(s) Spécifique(s)), el criterio del origen del público permite igualmente discriminar entre el FLP y el FS (Français de Spécialité), un aspecto sobre el que Mourlhon-Dallies insiste en "Penser le français langue professionnelle", artículo de ese mismo, 2006, pero que citaremos por su versión en línea de 2010, en el que la autora atribuye el nacimiento del FLP a una demanda concreta:

[...] une demande d'amélioration des compétences en français pour l'exercice des professions, émanant le plus souvent d'organisations professionnelles fédérées en réseaux (office de professions, fédérations de branches de métiers, regroupement d'écoles spécialisées). Aux plans politique et économique, ces nouvelles demandes résultent de l'intensification des flux migratoires à tous les niveaux de qualification. Elles concernent principalement des étudiants ou professionnels en fin ou en complément de spécialisation et des migrants venus travailler en France ou dans un pays francophone ${ }^{10}$.

Las divergencias entre los especialistas no se quedan aquí, y para comprobarlo basta con remitirse, por ejemplo, al manual Le Français sur objectifs spécifiques et la classe de langue (2007), de Carras, Tolas, Kholer y Szilagyi. Vuelven a aparecer aquí, aunque con ligeros cambios en la denominación, tres de las categorías de las que venimos hablando: "Français Objectif(s) Spécifique(s)" (FOS), "Français de Spécialité" (FS) y "Français Professionnel" (FP). Éste último, que en principio correspondería al anterior FLP, se encuentra ahora dividido en dos grupos, "Français à Visée Professionnelle" (FVP) y "Français Langue Professionnelle" (FLP), sobre los que se nos dice: 
Les appellations Français à Visée Professionnelle et Français Langue Professionnelle sont plus récentes, elles ne s'opposent pas aux appellations précédentes, elles les complètent plutôt. Le Français à Visée Professionnelle s'inscrit dans une perspective transversale aux différents champs de spécialité et secteurs d'activité : d'un point de vue didactique, cet enseignement vise à faire acquérir des compétences décloisonnées (communes à différents secteurs d'activité, à différents postes de travail), liées à la communication dans le monde professionnel [....]. L'enseignement de ces diverses compétences peut se faire auprès du public ayant un niveau très basique non seulement en langue française mais aussi dans leur (future) spécialité. [...]

L'appellation Français Langue Professionnelle concerne, quant à elle, la formation des publics en voie de spécialisation, en voie de professionnalisation, et témoigne donc d'une double exigence de formation, en français et dans le domaine professionnel (2007, pp. 19-20).

Por último, la tendencia a la atomización del francés asociado al ámbito profesional se prolonga el citado artículo de Sowa y Gajewska, "FS, FOS, FLP... Étiquettes vides ou concepts éducatifs opérationnels"11, en el que se propone una triple distinción entre FS (Français de Spécialité) y FCP (Français de la Communication Professionnelle) y FLP (Français Langue Professionnelle) que resulta novedosa: el FS sería, según ellas, la enseñanza de un sector preciso, el FCP la enseñanza a un público concreto y el FLP la mezcla de las dos anteriores.

Así las cosas, resulta cada vez más difícil tener una visión de conjunto de un campo tan diverso como el francés de especialidad. Por ello, y a modo de resumen, a continuación ofrecemos una tabla de sus principales modalidades, en la que se recogen, marcados en negrita, aspectos distintivos como la amplitud de los contenidos, la duración de la formación, el nivel de especialización del público al que van dirigido y el origen de la oferta:

\begin{tabular}{lllll}
\hline $\begin{array}{l}\text { Français } \\
\text { Spécialité (FS) }\end{array}$ & de & $\begin{array}{l}\text { Français } \\
\text { Objectif(s) } \\
\text { Spécifique(s) } \\
\text { (FOS) }\end{array}$ & $\begin{array}{l}\text { Français Langue } \\
\text { Professionnelle } \\
\text { (FLP) }\end{array}$ & $\begin{array}{l}\text { Français à Visée } \\
\text { professionnelle } \\
\text { (FVP) }\end{array}$ \\
\hline $\begin{array}{l}\text { Formación amplia } \\
\text { de contenidos de de contenidos de de contenidos de de contenidos de }\end{array}$ & $\begin{array}{l}\text { Formación precisa } \\
\text { un mismo dominio. }\end{array}$ & $\begin{array}{l}\text { Formación amplia } \\
\text { un solo dominio. }\end{array}$ & $\begin{array}{l}\text { un mismo dominio. } \\
\text { un mismo dominio. }\end{array}$ \\
\hline
\end{tabular}

11 Las autoras comparan además en este trabajo la realidad francesa con la de otras lenguas como el inglés, el español, el ruso, el alemán o el italiano para concluir que las diferentes denominaciones se basan en metodologías de enseñanza diferentes. 


\begin{tabular}{|c|c|c|c|}
\hline $\begin{array}{l}\text { Formación a medio } \\
\text { o a largo plazo. }\end{array}$ & $\begin{array}{l}\text { Formación a corto } \\
\text { plazo. }\end{array}$ & $\begin{array}{l}\text { Formación a medio } \\
\text { o a largo plazo }\end{array}$ & $\begin{array}{l}\text { Formación a medio } \\
\text { o a largo plazo }\end{array}$ \\
\hline $\begin{array}{l}\text { Público } \\
\text { especializado }\end{array}$ & $\begin{array}{l}\text { Público } \\
\text { especializado }\end{array}$ & $\begin{array}{l}\text { Publico en vías de } \\
\text { especialización }\end{array}$ & $\begin{array}{l}\text { Público } \sin \\
\text { especialización }\end{array}$ \\
\hline $\begin{array}{l}\text { Oferta de } \\
\text { formación de temas } \\
\text { y de competencias } \\
\text { ligadas a un sector } \\
\text { profesional }\end{array}$ & $\begin{array}{l}\text { Respuesta a } \\
\text { necesidades } \\
\text { específicas de un } \\
\text { sector profesional }\end{array}$ & $\begin{array}{l}\text { Oferta de } \\
\text { formación de temas } \\
\text { y de competencias } \\
\text { ligadas a un sector } \\
\text { profesional }\end{array}$ & $\begin{array}{l}\text { Oferta de } \\
\text { formación de temas } \\
\text { y de competencias } \\
\text { ligadas a varios } \\
\text { sectores } \\
\text { profesionales }\end{array}$ \\
\hline
\end{tabular}

Tabla 3. Aspectos distintivos de los diferentes campos del francés de especialidad.

Antes de cerrar este apartado, conviene recordar la razón por la que, a lo largo de este segundo apartado, y al contrario de lo que hicimos en el primero, no hemos hecho referencia a las metodologías de enseñanza: en el francés de especialidad en general, al igual que en todos los campos de adquisición de una lengua extranjera, la metodología viene claramente marcada por las directrices de MCERL. Es decir, los alumnos deben manejar, según el nivel de especialización, destrezas relacionadas tanto con la producción y comprensión oral como con la producción y comprensión escrita siempre en el marco del campo se especialidad elegido.

\section{4. Últimas tendencias en la enseñanza del francés}

No queremos cerrar este trabajo sin dedicar un breve apartado a dos de las tendencias más recientes que han aparecido en el ámbito educativo del francés: nos referimos al FOU (Français Objectif(s) Universitaire(s)), al FLS (Français Langue Seconde) y al FLI (Français Langue d'Intégration).

El FOU, del que se empieza a hablar a principios de la actual década ${ }^{12}$, es considerado de manera unánime por la crítica como una rama del $\mathrm{FOS}^{13}$. También en este campo serán Mangiante y Parpette quienes establezcan las bases teóricas de esta modalidad en Le français sur objectif universitaire (2011), que se ha convertido en el manual de referencia sobre la cuestión. Según señalan los autores, el FOU nace con el objetivo de dar

12 Cierto es que, ya en 2003, Tauzin hacía una diferenciación clara entre lo que él denomina "francés para trabajar" y "francés para estudiar". Sin llegar nunca a teorizar sobre esta diferencia, Tauzin avanzaba ya en cierta forma la necesidad de distinguir dentro del FOS una rama diferente destinada a un sector no profesional.

13 Así lo defienden por ejemplo Mangiante, Parpette o Mourlhon-Dallies en sus respectivos trabajos de 2011. 
respuesta a un público muy heterogéneo y sin embargo muy bien definido, ya que está formado por alumnos universitarios con intención de cursar sus estudios en Francia o en Universidades francófonas, con las necesidades comunicativas que ello supone. Pues bien, también en esta ocasión Mourlhon-Dallies ha venido a matizar la definición de sus colegas. En su artículo "Le français objectifs universitaires, entre français académique, français de spécialité et français préprofessionnel" (2011), la autora apela en primer lugar a la diversidad del público del FOU para defender el uso en plural de este término $(\text { FOUS })^{14}$. Seguidamente, y aunque no desmiente la estrecha relación que mantiene con el FOS, Mourlhon-Dallies defiende que el FOU posee igualmente una relación directa con otras metodologías como el FLS (Français Langue Séconde), el FIU (Français d'Intégration Universitaire) y el FS (Français de Spécialité), por lo que termina definiendo el FOU como el resultado de la siguiente ecuación: "FOU = FA+FLS+FIU + $x \%$ deFS [dérivé de FOS]" (2011, p. 137).

En su ecuación, Mourlhon-Dallies introduce dos nuevas disciplinas sobre las que se está centrando el debate acerca de la enseñanza del francés de los últimos años: nos referimos al FLS y al FLI. En este caso, el público recupera su papel discriminatorio, pues si el FLS tiene como objetivo principal la adquisición del francés por parte de los inmigrantes llegados a Francia en edad de escolarización, el FLI parece estar claramente orientado a los inmigrantes adultos en vías de incorporarse al mundo laboral. Aunque es un hecho que el fenómeno de la inmigración sigue marcando la evolución del francés de especialidad, carecemos de la distancia temporal que nos permita saber lo que darán de sí estas últimas incorporaciones, así como otras que, con toda seguridad, seguirán apareciendo: esperamos en cualquier caso que la revisión que hemos llevado a cabo hasta aquí sea de ayuda en el futuro, cuando sea tiempo de juzgar el alcance de cada una de ellas dentro del universo educativo.

\section{About the author}

Elena Cuasante Fernández es profesora asociada en la Universidad de Cádiz desde 1998 y doctora desde 2005. En la actualidad pertenece a diferentes grupos de Investigación dedicados a la literatura francófona y la difusión del francés como lengua extranjera. En los últimos años ha centrado su docencia e interés investigador en el francés para fines específicos. Profesora durante años de las asignaturas "Francés

No se trata de una novedad ya que la autora defendió igualmente la descomposición de FOS en plural y no en singular. 
aplicado a la gestión de empresas" y "Francés aplicado a las finanzas y a la contabilidad" en la Facultad de Ciencias Económicas y Empresariales de la Universidad de Cádiz, en la actualidad imparte y coordina un módulo de francés sectorial en el Grado de Estudios Franceses así como la asignatura de "Técnicas de comunicación y traducción francés/español para las transacciones comerciales y las gestiones administrativas internacionales" dentro del programa de Master en Comunicación Internacional de la Universidad de Cádiz. Ha dirigido igualmente diferentes trabajos de Fin de Grado y de Fin de Master sobre cuestiones relacionadas con el francés para fines profesionales, a saber: "El francés sectorial y su traducción: análisis de un caso práctico", "La comunicación comercial en el ámbito francófono" o "El francés de especialidad y sus ámbitos".

\section{Article history}

Paper received: 8th February 2017

Paper received in revised form and accepted for publication: 29th October 2017

\section{Referencias bibliográficas}

Aupècle, M. y Alvarez, G. (1977). Français instrumental/français fonctionnel. Montréal: AUPELF.

Cadet, L. y Guerin, E. (2012). FLM, FLE, FLS, au-delà des catégories. Le français aujourd'hui ,146, 3-8. < $\quad$ http://www.cairn.info/revue-le-francais-aujourd-hui-2012-1-page-3.htm> [30/01/2016].

Carras, C., Tolas, J., Kholer, P. y Szilagyi, É. (2007). Le français sur objectifs spécifiques et la classe de langue. Paris: CLE International.

Cuq, J-P. (2003). Dictionnaire de didactique du français langue étrangère et seconde. Paris: CLE International.

Gueorguieva-Steenhoute, E. (2008). La langue de spécialité est-elle soluble dans le FLE, le FOS et le FLP? En D. De Vecchi y Cl. Martinot (Eds.), Les langues de spécialité en question : perspectives d'étude et applications. Actes de la 12éme Journée Scientifique de la CRL (pp. 33-40). Universidad Paris-Diderot.

Hall, E. T. y Reed Hall, M.R. (1990). Understanding Cultural Differences: Germans, French and Americans. London: Nicholas Brealey Publishing.

Lehmann, D. (1993). Objectifs spécifiques en langues étrangères. Paris: Hachette.

Lehmann, D. (1996a). Le français sur objectifs spécifiques aujourd'hui: une approche 
délinéarisée, linguistique et culturelle. L'avenir du français dans une Europe pluriculturelle. Actas del $2^{\circ}$ Congreso Nacional de profesores de Francés (pp. 219-221). Universidad de Aristote, Thessalonique.

Lehmann, D. (1996b). Le français sur objectifs spécifiques et l'intégration de la composante culturelle. L'avenir du français dans une Europe pluriculturelle. Actas del $2^{\circ}$ Congreso Nacional de profesores de Francés (pp. 224-228). Universidad de Aristote, Thessalonique.

Mangiante, J-M. y Parpette, Ch. (2004). Le français sur objectif spécifique: de I'analyse des besoins à l'élaboration d'un cours. Paris: Hachette FLE.

Mangiante, J-M. (2006). Français de spécialité ou français objectif spécifique: deux démarches didactiques distinctes. En J. Pinilla Martinez, M.E. Jiménez, F. Olmo Cazevieille, G. Peña Martínez, M. López y I. Tamarit Vallés (Coords.), Linguistique plurielle. Congreso Internacional de lingüística francesa (pp. 137-152). Valencia : Editorial UPV.

Mangiante, J-M. y Parpette, Ch. (2011). Le français sur objectif universitaire. Paris: PUG.

Moirand, S. (1993). Décrire les discours de spécialité. En S. Barruecos, E. Hernández y L. Sierra (Eds.), Actas de las III Jornadas de lenguas para fines específicos (pp. 15-17). Madrid: Servicio de Publicaciones Universidad de Alcalá.

Mourlhon-Dallies, F. (2006). Du français sur Objectifs spécifiques au français Langue Professionnelle. Actas de Séminaire de réflexion. Centre international d'études pédagogiques. (pp. 28-33). Paris : Ministère de la Culture et de la Communication.

Mourlhon-Dallies, F. (2010). Penser le français langue professionnelle. Le Français dans le Monde, 346, 25-28. <http://www.fdlm.org/fle/article/mourlhon.php> [30/01/2016].

Mourlhon-Dallies, F. (2011). Le français objectifs universitaires, entre français académique, français de spécialité et français préprofessionnel. <http://www.culturecommunication.gouv.fr/Ressources/Comptes-rendus-de-colloquesjournees-d-etude/Recontres-2006-apprendre-le-francais-dans-un-contexte-professionne [30/01/2016].

Porcher, L. (1976). Mr. Thibault et le bec bunsen. Études de linguistique appliquée , 23, 6-13.

Qotb, H. (2008). Vers une didactique du Français sur Objectifs Spécifiques médié par Internet. Paris : Les Éditions Publibook.

Richer, J.-J. (2007). Le FOS est-il soluble dans le FLE? Pour une didactique spécialisée du Français sur Objectifs spécifiques (F.O.S.). En D. De Vecchi, Dardo y C. Martinet (Eds.), Les langues de spécialité en question : perspectives d'étude et appli

cations, Publications en ligne de la CRL $n^{\circ} 1$. 〈https://gerflint.fr/Base/Perou2/Richer.pdf> [30/01/2016]

Sowa, M. y Gajewska, E. (2013). FS, FOS, FLP... Etiquettes vides ou concepts éducatifs opérationnels ? Points Communs, 1, 18-28.

Tauzin, B. (2003). Outils et pratiques du FOS dans l'enseignement, la formation d'enseignants, les examens et l'édition, in $Y$-a-t-il un français sans objectif(s) spécifique(s) ? Les cahiers de 
l'Asdifle, 14, 71-83.

Zolana, M. A. (2013). Concevoir un programme de Français sur Objectifs Spécifiques. Difficultés théoriques et pratiques: le cas de la faculté d'économie de l'université Agostinho Neto Luanda-Angola, Thèse de Doctorat en Sciences du langage: Didactique des langues étrangères et secondes Université de Nice Sophia-Antipolis. 